\title{
Análise da expansão do cultivo da palma de óleo no Nordeste do Pará
}

\section{Analysis of palm oil expansion in Eastern Pará}

Wanja Janayna de Miranda Lameira. Geógrafa - Doutorando em Ciências Ambientais; Departamento de Geociências/Universidade Federal do Pará - UFPA. E-mail: wjlameira@hotmail.com

Ima Célia Guimarães Vieira - Agrônoma. Pesquisadora do Museu Paraense Emilio Goeldi - MPEG - Belém, Pará. E-mail: ima@museu-goeldi.br

Peter Mann de Toledo - Biólogo.Pesquisador do Instituto Nacional de Pesquisas Espaciais - INPE, São Paulo. E-mail: peter.toledo@hotmail.com

\section{Resumo}

A palma de óleo (Elaeis guineensis Jacq.) ganhou maior visibilidade e incentivos na região nordeste da Amazônia nos últimos dez anos e um conjunto de ações políticas recentes permitiram a formação de territórios da palma. O objetivo deste trabalho é analisar a expansão dessa cultura no período 2008 a 2013, na região conhecida como polo do dendê no Pará, utilizando Sensoriamento Remoto e Sistema de Informação Geográfica. O polo está localizado no nordeste do estado e abrange cerca de $59.600 \mathrm{~km}^{2}$ e 37 municípios. Os resultados mostram a existência de dois padrões espaciais bem distintos: (i) áreas de cultivo de palma de óleo com mais de 10 anos de produção e presença de menor número de empresas; (ii) áreas recentes com palma de óleo, presença de maior número de empresas e melhores condições de infraestrutura.O contexto em que se expande a produção da palma no Pará é de vulnerabilidade socioeconômica e desafia a produção sustentável da cultura no estado.

\section{Palavras-chave}

Dendeicultura, geotecnologias, sustentabilidade.

\begin{abstract}
The oil palm (Elaeis guineensis Jacq.) plantation has gained special attention and political incentives in the northeast region of the Amazon in the past decade through a set of concerted governmental actions that have resulted in the formation of extensive palm oil territories. The objective of this study is to analyze the expansion of this crop in the period between 2008 and 2013, in the region known as 'Polo do Dendê' in the State of Pará, using remote sensing and Geographic Information System techniques. This region is located in northeastern of Para state and covers about 59,600 square kilometers and encompass 37 municipalities. The results show the existence of two distinct spatial patterns: (i) oil palm growing areas with more than 10 years of production and presence of a handful of oil extraction companies, and; (ii) recent areas with oil palm expansion, with the presence of a higher number of companies and better infrastructure conditions. The context in which expands the palm oil production in Pará is associated to a socioeconomic vulnerability and challenges the sustainable production of this crop in the state.
\end{abstract}

\section{Keywords}

Palm oil, geotechnologies, sustainability. 


\section{INTRODUÇÃO}

A região amazônica ganha protagonismo no cenário internacional por sua grande extensão de áreas com floresta tropical e alta diversidade biológica e sócio-cultural. Em contrapartida, também vivencia a expansão das áreas urbanas e a intensificação das atividades agropecuárias, principalmente, a partir da década de 1970, com a implantação de projetos governamentais de desenvolvimento e de disputas territoriais, introduzindo novas relações sociais, econômicas, políticas, além de inúmeros problemas ambientais.

Nos últimos dez anos, a palma de óleo (Elaeis guineensis Jacq.) ganhou maior visibilidade e incentivos na região nordeste da Amazônia com o zoneamento do cultivo e com o aumento de demanda mundial pelo óleo (BECKER, 2010). Estabeleceu-se uma política nacional dos biocombustíveis como estratégia governamental para enfrentar a crise energética mundial e atender aos acordos internacionais assumidos pelo Brasil.

A matriz da bioenergia implantada na região amazônica, caracterizada por estratégias "verdes", "sustentáveis" ou "ecológicas", legitimam a implantação da dendeicultura na região (BACKHOUSE, 2013), reestruturam as práticas da agricultura familiar e intensificam as transformações sociais e os conflitos territoriais (NAHUM \& MALCHER, 2012).

Assim, a atual dinâmica socioespacial do nordeste paraense é marcada pela expansão da dendeicultura e um conjunto de ações políticas estatais e empresariais que permitiram a formação de territórios da palma ( NAHUM \& SANTOS, 2015).

Neste trabalho, analisa-se a expansão da palma de óleo no nordeste do estado do Pará (polo do dendê), no período de 2008 a 2013 com o intuito de contribuir para o entendimento das mudanças de usos da terra e das recentes transformações que ocorrem no território paraense.

\section{METODOLOGIA}

\section{1 ÁREA DE ESTUDO - POLO DO DENDÊ, PARÁ}

Há duas propostas de delimitação do polo do dendê no Estado do Pará. A primeira (aproximadamente $46.900 \mathrm{~km}^{2}$ ) foi definida por Bastos (2001) para se referir às áreas com grande potencial de produção, levando em consideração os riscos climáticos a essa cultura. A segunda, (polo de produção de biodiesel- cerca de $59.600 \mathrm{~km}^{2}$ ), criada no âmbito do programa do Biodiesel (MDA, 2010), considera as condições edafoclimáticas, priorizando, contudo, a expansão da palma de óleo em áreas que já tiveram a cobertura florestal primária alterada (desflorestada). 
Para esse estudo, adotou-se o recorte espacial do MDA (2010) (Figura 1), por indicar as áreas para onde a dendeicultura poderá expandir futuramente. Este polo abrange 37 municípios da região nordeste paraense (IBGE, 2014), apontados como prioritários para investimentos da dendeicultura no Estado (SILVA et al., 2014).

Figura 1 - Localização da área de estudo no nordeste do estado do Pará.

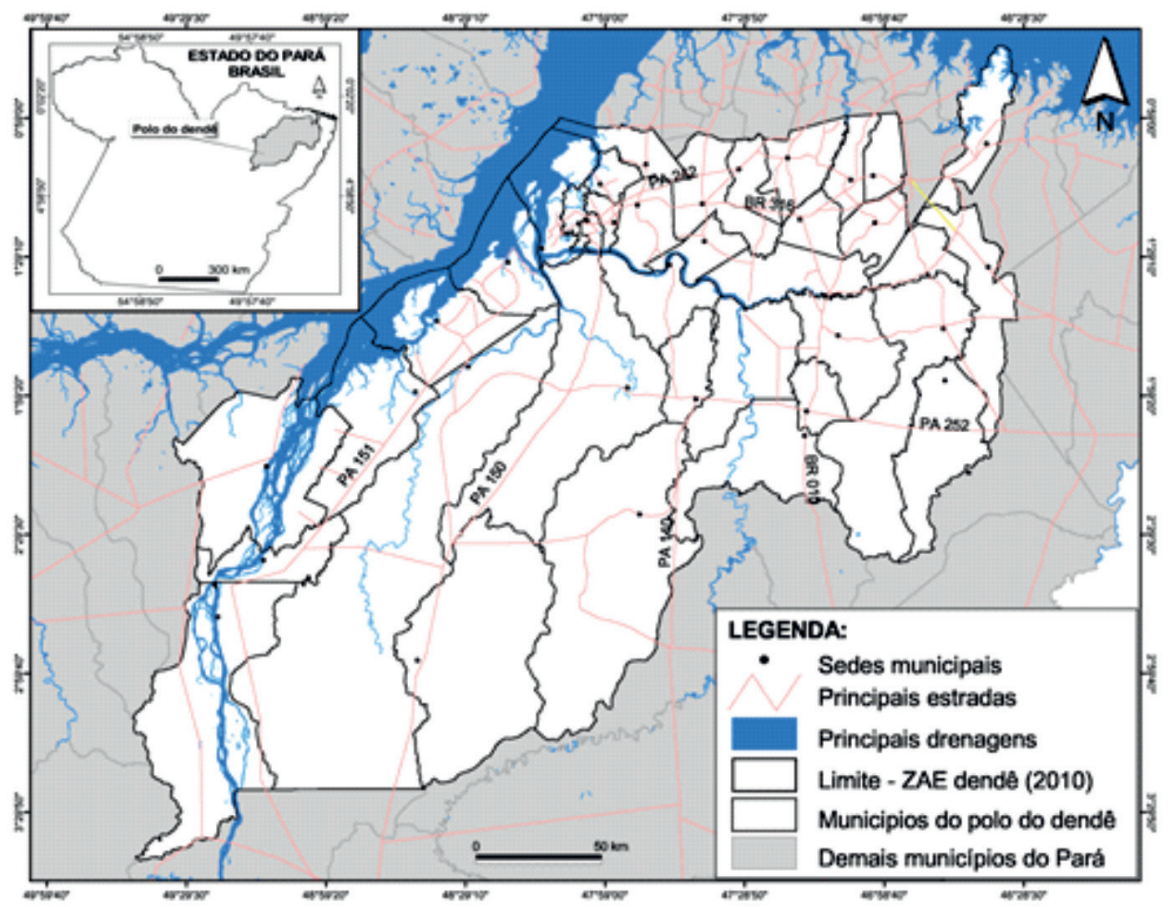

Fonte: Adaptado do MDA (2010).

De acordo com De Souza e Rocha (2014), o padrão climático do nordeste do Pará é definido, principalmente, pela Zona de Convergência Intertropical ZCIT, as linhas de instabilidades provocadas pelas brisas marítimas e fluviais e pelo aquecimento local. A temperatura apresenta oscilações entre $26,0^{\circ} \mathrm{C}$ e $27,0^{\circ} \mathrm{C}$; a média anual de precipitação pluviométrica anual é de aproximadamente 2.522,6 mm (1983 a 2010) (De SOUSA et al., 2010).

Monteiro et al. (2009) descrevem que a compartimentação geológica é formada por sedimentos pós-barreiras e pelas Formações Barreiras e Ipixuna; o relevo é pouco acidentado, com a presença de baixos platôs aplainados, terraços e várzeas com amplitude altimétrica que variam de $14 \mathrm{~m}$ a $96 \mathrm{~m}$. Os solos predominantes são os Latossolos Amarelo distrófico (LAd) (EMBRAPA, 2009). 
A cobertura vegetal é formada pela Floresta Densa dos Baixos Platôs, a Densa de Platôs, bastante alterada, favorecendo o surgimento das Florestas Secundárias ou capoeiras (IBGE, 2012).

A população é formada, principalmente, por pequenos agricultores, ribeirinhos e quilombolas, que usam a terra como núcleo estruturante das comunidades (NAHUM e MALCHER, 2012). Baseado na classificação das trajetórias tecnológicas camponesas de Costa (2012), no pólo do dendê, observa-se basicamente dois padrões distintos: (i) a produção agropecuária, com predomínio da pecuária de corte; e o uso de sistemas agroflorestais, caracterizado por culturas temporárias e permanentes e a extração de produtos não madeireiros.

\subsection{CRIAÇÃO DO BANCO DE DADOS}

O mapeamento das áreas com palma de óleo foi desenvolvido nas plataformas do TerraView 4.2.2 (INPE) e QGis 2.10.1 para a elaboração do banco de dados, análise e consultas espaciais; e no ENVI 5.0 para a visualização, tratamento e análise dos dados de sensoriamento remoto.

Foram adicionados ao banco de dados as informações da área ocupada com palma de óleos em 2008 (ALMEIDA et al., 2014); as bases cartográficas de estradas, sedes municipais e limites municipais (IBGE, 2014); as imagens de satélite landsat 8, sensor OLI (USGS, 2013); e os pontos de GPS obtidos na expedição de campo em 2013. O sistema de projeção adotado foi o latlong, Datum WGS 84.

\section{3 MAPEAMENTO DAS ÁREAS DE PLANTIO DE PALMA DE ÓLEO}

Neste trabalho, as áreas plantadas com palma de óleo tiveram duas fontes de dados: (i) o banco de dados do Centro de Endemismo Belém (ALMEIDA et al.; 2014), para o ano de 2008 e (ii) a classifificação de imagens orbitais do sensor 8/OLI (Oitava geração do Landsat com resolução espacial de $30 \mathrm{~m}$.

Para a classificação das imagens, utilizou-se as órbitas/ponto 223/061, 223/062, 224/061, 224/062 e 224/063, obtidas entre os meses de julho e agosto de 2013 e aplicou-se o realce pelo método de aumento linear, procedimento que ajusta as barras dos níveis de cinza igualmente distribuídos pelo histograma, ampliando o contraste na cena para melhorar a interpretação visual (GOEL, 1989).

Com o auxílio do banco de dados do Centro de Endemismo Belém, foi processada a classificação supervisionada na composição colorida 4 (B), 5 (G) e 6 (R). O algoritmo adotado foi o algoritmo de vizinhança próxima (Maximum Likelihood), que considera a ponderação das distâncias das médias e utiliza parâmetros estatísticos para escolher a classe de um pixel (MYUNG, 2003). 
Para a validação dos resultados da classificação de 2013, foram utilizados a Matriz de Erro e o índice Kappa (HUDSON e RAMM, 1987). Ambos os métodos têm sido utilizados para avaliar a exatidão do processo de classificação automatizada das imagens de satélite (CAMPBELL, 1987).

Sabe-se que os erros estão presentes no processo de classificação automatizada decorrentes das interações complexas entre as estruturas da paisagem, o algoritmo utilizado e a resolução do sensor, entre outros. Contudo, a forma padronizada de reportar os erros em locais específicos é mediante o uso da Matriz de erro ou Matriz de confusão, que identifica não somente a Exatidão Global, mas também os erros de inclusão e omissão para cada uma das classes temáticas trabalhadas (CONGALTON, 1991). Já o índice de Kappa é uma medida empregada para avaliar a exatidão de todos os elementos da matriz de erro, ou seja, analisa em conjunto todos os elementos da matriz, ao invés de apenas os valores que estão situados na diagonal da mesma, o que ocorre quando se calcula a exatidão global (ROSENFIELD e FITZPATRICK-LINS, 1986).

\subsection{EXPEDIÇÕES DE CAMPO}

O reconhecimento da área de estudo e a validação da classificação supervisionada de 2013 foram realizados, respectivamente, em duas viagens de campo, uma em setembro de 2011 e outra em novembro de 2013. Para a validação das áreas com palma de óleo foram checados em campo 468 pontos (selecionados aleatoriamente para a área de estudo). Os municípios visitados foram Mocajuba, Cametá, Abaetetuba, Santa Bárbara do Pará, Santo Antônio do Tauá, Igarapé-Açu, Bonito, São Miguel do Guamá, São Domingos do Capim, Bujaru, Concórdia do Pará, Tomé-Açu, Tailândia, Moju e Acará.

\subsection{ANÁLISE ESPACIAL}

A análise espacial em ambiente Sistemas de Informações Geográficas (SIG) está fortemente relacionada com os modelos de representação de dados geográficos (QUEIROZ FILHO \& MARTINELLI, 2007), sendo que a forma mais importante de análise de dados proporcionada pelo modelo de campos é a álgebra de mapas ou modelagem cartográfica (TOMLIN, 1998). Assim, neste estudo, a análise espacial da palma de óleo em 2008 e 2013 foi realizada utilizadose a álgebra de mapas e o tratamento estatístico dos dados. 


\section{RESULTADOS E DISCUSSÃO}

\subsection{ACURÁCIA DOS DADOS}

A Tabela 1 apresenta a matriz de erro, na qual se observa a análise de 468 pontos obtidos na expedição de campo de 2013.

Tabela 1. Matriz de erro da classificação supervisionada de 2013.

\begin{tabular}{l|c|c|c|c|c}
\hline \multirow{2}{*}{$\begin{array}{l}\text { Classificação } \\
\text { supervisionada }\end{array}$} & \multicolumn{5}{|c}{ Expedição de campo (novembro 2013) } \\
\cline { 2 - 6 } & $\begin{array}{c}\text { Palma de } \\
\text { óleos }\end{array}$ & Outros & Total & $\begin{array}{c}\text { Erro de Inclusão } \\
(\%)\end{array}$ & $\begin{array}{c}\text { Acurácia } \\
(\%)\end{array}$ \\
\hline 1. Palma de óleos & 323 & 9 & 332 & 2,71 & 97,29 \\
\hline 2. Outros & 11 & 125 & 136 & 8,09 & 91,91 \\
\hline Total de pixels de campo & 334 & 134 & 468 & - & $\begin{array}{c}\text { Exatidão } \\
\text { Global } \\
94,60\end{array}$ \\
\hline Erro de Omissão (\%) & 3,29 & 6,72 & - & - & - \\
\hline
\end{tabular}

Fonte: Organização dos autores, (2015).

Do total de pontos avaliados, 448 foram classificadas corretamente. Os erros de inclusão e omissão foram maiores na classe "outros" (8,09\% e 6,72\%, respectivamente). A acurácia de 97,29\% das áreas de palma de óleo se deve, principalmente, à utilização da forma geométrica e da textura na identificação dessas áreas (principalmente as grandes áreas). Contudo, nas regiões com pequenos plantios (como os encontrados nos municípios de Mocajuba, Cametá e Abaetetuba), a identificação foi baixa, devido à semelhança do comportamento espectral dos palma de óleos, por exemplo, com a vegetação secundária.

No geral, a Exatidão Global das áreas de palma de óleos em 2013 foi de 94,60\% e o Índice Kappa foi de 0,89. Baseados em Hudson e Ramm (1987), valores acima de $80 \%$ obtidos em uma classificação de imagem de satélite podem ser considerados excelentes.

\subsection{EXPANSÃO DO PLANTIO DE PALMA DE ÓLEO DE 2008 A 2013}

Em 2008, identificou-se nas imagens de satélite, que os cultivos de palma de óleo ocupavam uma área de aproximadamente 80.272 hectares, o que representa $1,35 \%$ da área total do Polo do dendê. Neste ano, observou-se a existência de duas áreas onde estão concentrados os plantios de palma de óleo (Figura 2). 
Figura 2 - Distribuição dos plantios de palma de óleosem 2008 no nordeste do estado do Pará (Polo do Dendê).

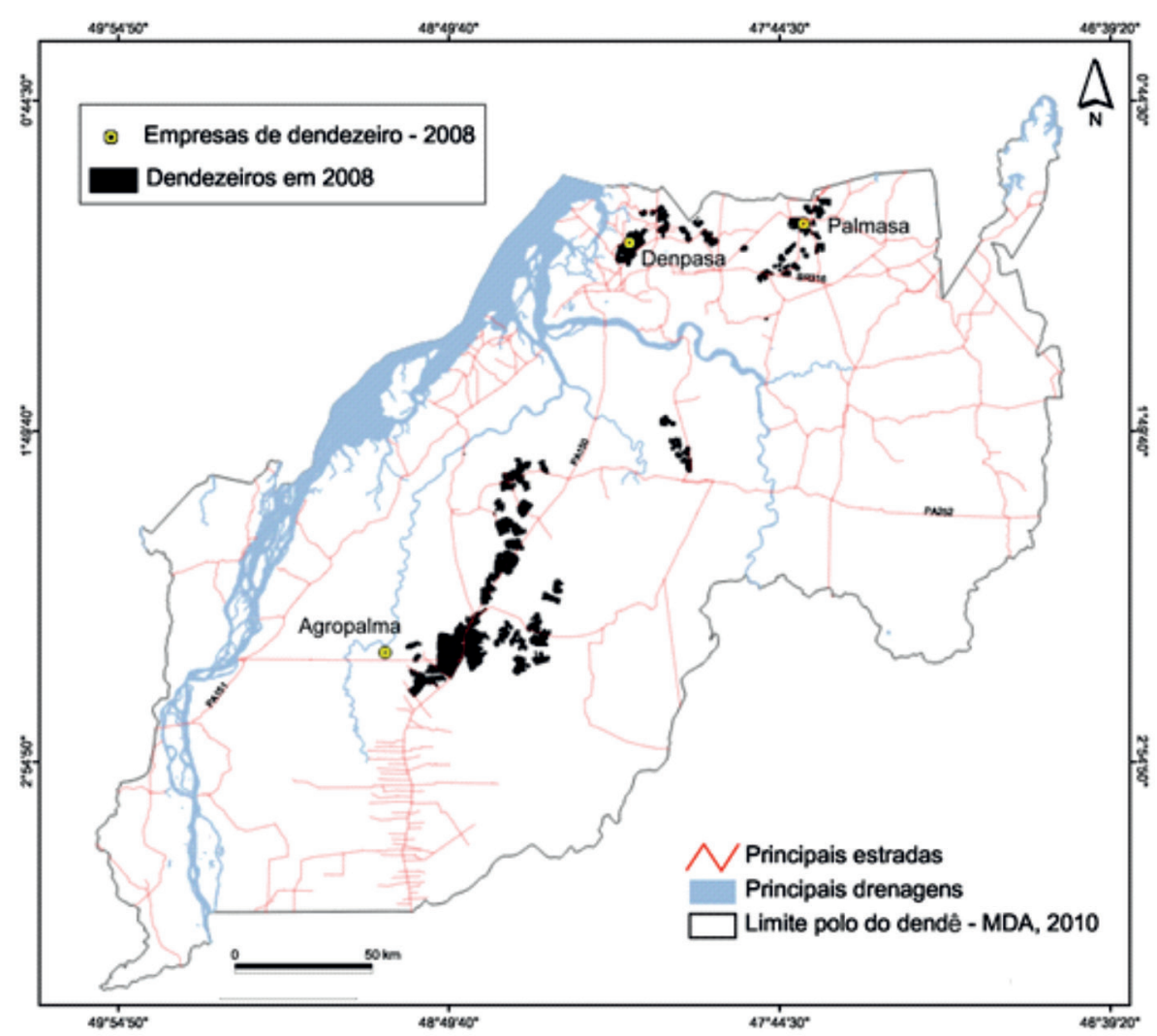

Fonte: Adaptado de Almeida et al. (2014).

A primeira está localizada na porção centro-oeste da área de estudo, às proximidades dos municípios de Acará, Moju e Tailândia, que juntos possuem as maiores áreas com ocupação de palma de óleo e têm como principal empresa de atuação, nesse período, a empresa Agropalma. A segunda está localizada mais ao norte da área de estudo, às imediações dos municípios de Santa Bárbara, Santo Antônio do Tauá e Igarapé-Açu, tendo como principais empresas atuantes a Denpasa, Dentauá e a Palmasa, respectivamente.

Em 2013, há um aumento das áreas com cultivos da palma (de 80.272 ha para $146.611 \mathrm{ha}$ ) e a presença de um maior número de empresas que passam a atuar no polo do dendê (Figura 3). 
Figura 3 - Distribuição dos plantios de palma de óleo em 2013 no nordeste do estado do Pará (Polo do Dendê).

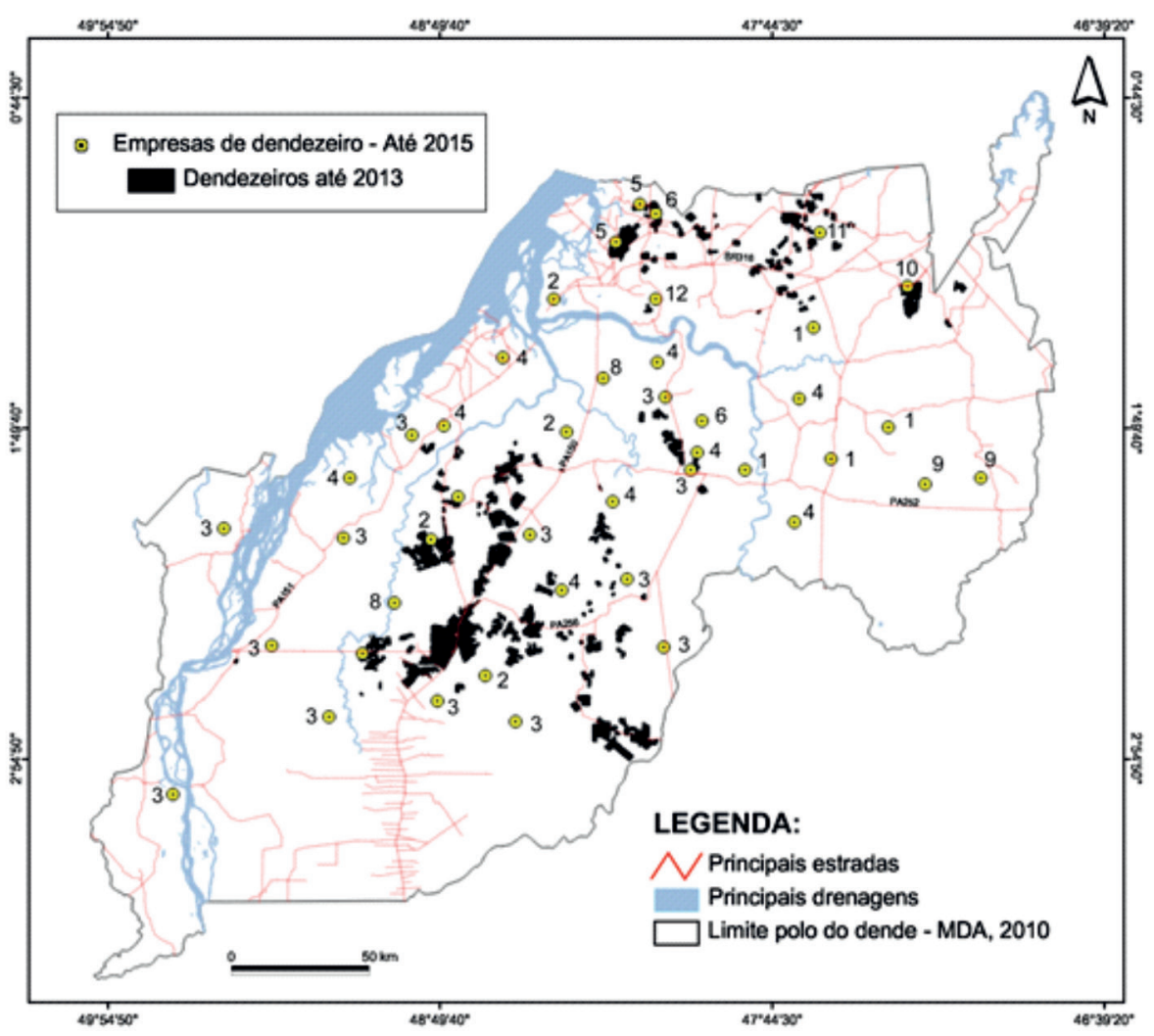

* Principais empresas: 1. ADM; 2. Agropalma; 3. BBB/Petrobras/Galp; 4. Biopalma; 5. Denpasa; 6. Dentauá; 7. Granfeng Group; 8. Marborges; 9. Mejer; 10. Palmasa; 11. Yossan

Fonte: Trabalho de campo (2013) e Nahum e Santos, (2015).

A localização preferencial dos monocultivos são os municípios de Moju, Tailândia, Acará e Tomé-Açu, onde estão concentradas as maiores áreas e as três maiores empresas do ramo (Agropalma, Biopalma e BBB/Petrobras/Galp, que possuem propriedades próprias e em parceria com agricultores camponeses da região). Outras áreas, como São Domingos do Capim, Bujaru, Concórdia do Pará, Igarapé-Açu e Bonito, que antes dessa política eram quase inexpressivos, em 2013 a palma passa a ocupar um maior espaço.

Em síntese, de 2008 a 2013 é possível distinguir dois padrões espaciais: (i) o menor número de empresas e áreas com cultivo de palma de óleos com mais de 10 anos de produção; (ii) o maior número de empresas, melhores condições de infraestrutura e novos plantios de palma de óleos (Figura 4). 
Figura 4 - Distribuição dos plantios de palma de óleos em 2008 e 2013 no nordeste do estado do Pará (Polo do Dendê)

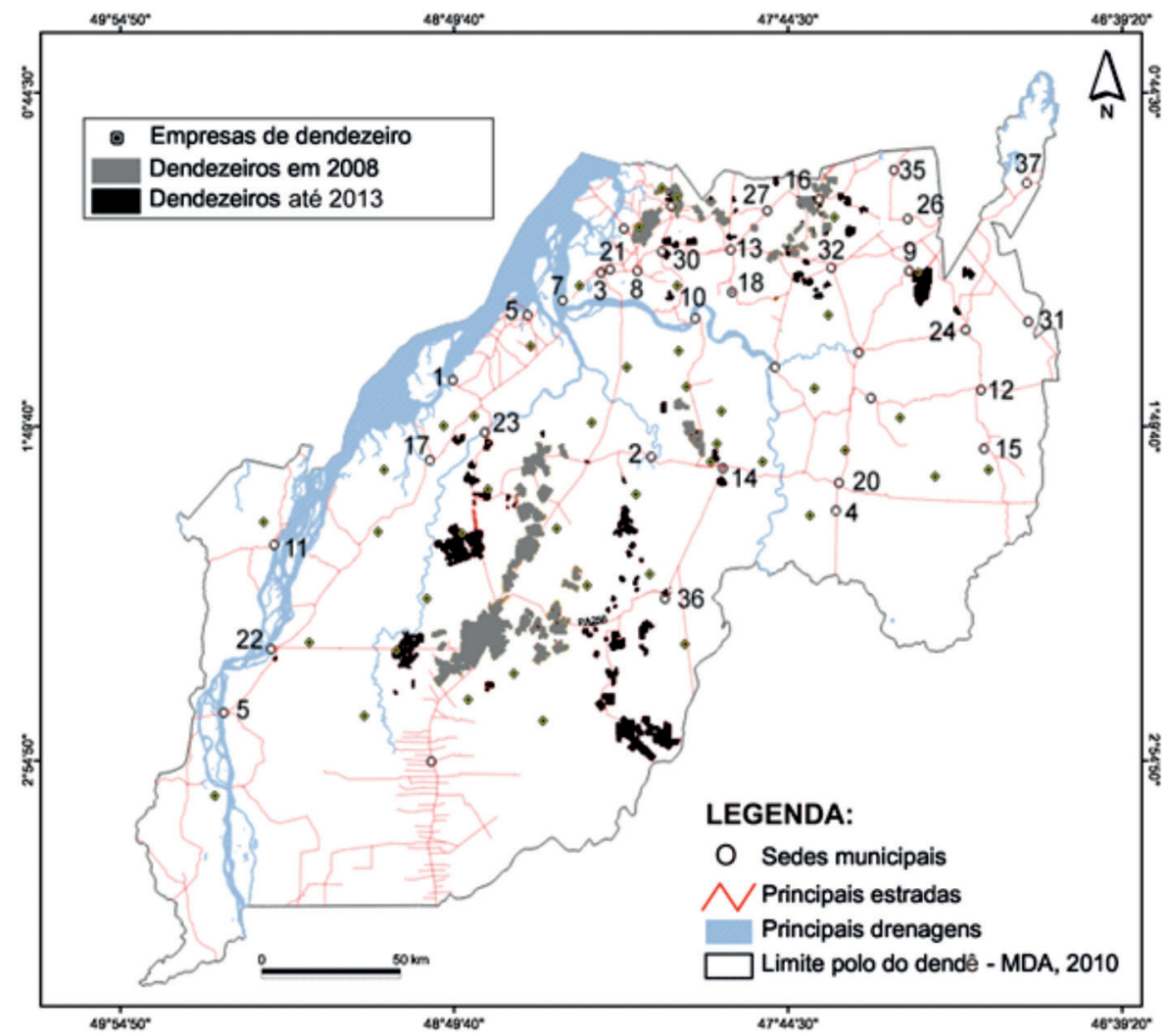

LEGENDA:

* Municípios: 1. Abaetetuba; 2. Acará; 3. Ananindeua; 4. Aurora do Pará; 5. Baião; 6. Barcarena; 7. Belém; 8. Benevides; 9. Bonito; 10. Bujaru; 11. Cametá; 12. Capitão Poço; 13. Castanhal; 14. Concórdia do Pará; 15. Garrafão do Norte; 16. Igarapé-Açu; 17. Igarapé-Miri; 18. Inhangapi; 19. Irituia; 20. Mãe do Rio; 21. Marituba; 22. Mocajuba; 23. Moju; 24. Nova Timboteua; 25. Ourém; 26. Peixe-Boi; 27. Santa Bárbara; 28. Santa Isabel do Pará; 29. Santa Luzia do Pará; 30. Santa Maria do Pará; 31. Santo Antônio do Tauá; 32. São Domingos do Capim; 33. São Francisco do Pará; 34. São Miguel do Guamá; 35. Tailândia; 36. Tomé-Açu; 37. Tracuatueua.

Fonte: Organização dos autores (2015).

De uma forma geral, observa-se a maior fluidez e conexões para produção e escoamento da produção de óleo de palma, devido principalmente a implantação da hidrovia do Capim, a duplicação, pavimentação e/ou restauração de estradas federais (BR 163), estaduais (PA 124; PA 140; PA 252; PA 253 e a PA 256), e a conclusão do sistema de transporte integrado do Pará - Alça Viária (CRUZ e ROCHA, 2005); além da abertura de linhas de credito (Pronaf Eco-Dendê), o 
Zoneamento Agroecológico da cultura da palma de óleo - ZAE; o programa Terra Legal, entre outros. No caso das empresas, sua atuação é mais perceptível a partir do aumento das áreas com plantios e a implantação de indústrias de extração e beneficiamento localizadas nos municípios do polo do dendê.

\section{CONSIDERAÇÕES FINAIS}

O aumento das áreas com palma de 2008 a 2013 no polo do dendê do Pará está fortemente ligado à política do governo federal (Programa Nacional de Produção e Uso de Biodiesel - PNB em 2004; o Programa de Produção Sustentável de Óleo de Palma - PPSOP em 2010), para a criação da matriz energética dos biocombustíveis. Esta ação do Estado em parceria com as empresas tem intensificado a verticalização da cadeia dendeícola, e promovido uma mudança do padrão de uso da terra na região, tornando-a mais dinâmica e conectada com o mercado do óleo de palma. Acompanhar as mudanças no território do dendê no Pará não é tarefa fácil, dada à complexidade social e de produção associada a uma dinâmica global.

A identificação e seleção de indicadores de sustentabilidade poderá contribuir para uma avaliação dos impactos socioambientais da expansão da palma em diferentes cenários de governança. Em maio de 2010, o governo do Pará e as empresas produtoras de dendê assinaram um Protocolo Socioambiental para a Produção de Óleo de Palma, com o objetivo de pactuar o compromisso de ambos com os princípios e critérios de sustentabilidade econômica e socioambiental da expansão da cadeia de produção da palma no estado. No entanto, uma análise inicial feita por Monteiro (2013) mostrou que diferentes sistemas produtivos com palma implantados no Pará, não têm observado as políticas públicas e as exigências do cadastramento ambiental da propriedade rural e o cumprimento do licenciamento da atividade rural, que devem garantir a recuperação das Áreas de Preservação Permanente e Reserva Legal, e promover a formação de corredores ecológicos, protegendo áreas de relevante interesse para a conservação. Em 2014, houve repactuação de ações conjuntas entre o poder público e o setor privado em um Protocolo de Intenções Socioambiental da Palma de óleo, muito mais amplo e frágil que o anterior.

O desafio é o de que a palma de óleo não amplie o desmatamento. Sua expansão está sendo feita, em grande parte, em áreas já desmatadas e degradadas, porém, como o programa brasileiro não especifica os níveis de degradação da terra e não apresenta uma definição oficial de degradação, e nem o mapeamento das terras degradadas, áreas em processo de regeneração e com alto valor de 
conservação da biodiversidade estão sendo usadas para a expansão da cultura (Almeida, A. comunicação pessoal). Além disso, nota-se a rápida formação de um mercado de terras nos municípios e a concentração de renda acentuada.

Outro aspecto a considerar na expansão da palma, é o cenário de sustentabilidade nos municípios do polo do dendê. Não há nada que limite a expansão do dendê em municípios cujas cadeias produtivas alimentares estejam consolidadas. O ideal seria que estes municípios limitassem o plantio do dendê em 10\% da área agrícola, como ocorre em municípios goianos onde a canade-açúcar se expande. Em geral, os municípios do polo possuem padrões de desenvolvimento diferenciados, vulnerabilidade socioeconômica e problemas socioambientais acentuados (LAMEIRA et al., 2015), além de fraca capacidade operacional da gestão ambiental (CARDOSO et al., 2013), que desafiam a expansão sustentável da palma de óleo.

\section{AGRADECIMENTOS}

Os autores agradecem ao apoio do INCT/ Biodiversidade e Uso da Terra na Amazônia (processo CNPQ no 574008/2008-0), pelo financiamento para a realização dos trabalhos de campo, e à Fundação Coordenação de Aperfeiçoamento de Pessoal de Nível Superior - CAPES, Brasil pela concessão da bolsa de estudo (Doutorado); e ao Programa de pós-graduação de Ciências Ambientais da Universidade Federal do Pará.

\section{REFERÊNCIAS}

ALMEIDA, A. S. de; VIEIRA I. C. G; ROCHA, D. di P. N. da. Caracterização e mapeamento dos padrões de uso e cobertura da terra na área de endemismo Belém. Museu Paraense Emílio Goeldi, 2014, 170p. (Relatório executivo).

BACKHOUSE, M. A desapropriação sustentável da Amazônia: caso de investimentos em dendê no Pará, Fair Fuels? Working Paper 6, Berlin. 2013, 31p.

BASTOS, T. X. Zoneamento de riscos climáticos para a cultura do palma de óleo no estado do Pará. Revista Brasileira de Agrometeorologia. Passo Fundo, v.9, n.3. 2001, p.564-570.

BECKER, B. K. Recuperação de áreas desflorestadas da Amazônia: será pertinente o cultivo da palma de óleo (dendê)?. Revista Confins. 2010,p. Disponível em: <https:// confins.revues.org/6609?lang=pt>. Acesso em: Jan./2012.

CAMPBELL, J. B. Introduction to remote sensing. New York, The Guilford Press, 1987. $551 \mathrm{p}$. 
CARDOSO; A. S.; TOLEDO, P. M. de; VIEIRA, I. C. G. Dimensão institucional da sustentabilidade e gestão ambiental no município de Moju, Pará: uma aplicação do Barômetro da Sustentabilidade. Sustentabilidade em Debate, Brasília, v. 5, n. 1, jan./ abr. 2014, p. 117-135.

CONGALTON, R. A Review of Assessing the Accuracy of Classification Remotely Sensed Data. Remote Sensing of Environment. USA, No 37. 1991, p. 35-36.

COSTA, F. de S. Mercado de terras e trajetórias tecnológicas na Amazônia. Economia e Sociedade, Campinas, v. 21, n. 2, p. 245-273, 2012.

CRUZ, B. E. V da; ROCHA, G. De M. Dendê como projeto de Estado: uma alternativa econômica, social e ecológica para a Amazônia. Observatório geográfico da América Latina. Observatório geográfico da America Latina, 2005, 19p. Disponível em: http://observatoriogeograficoamericalatina.org.mx/egal11/Geografiasocioeconomica/ Geografiapolitica/02.pdf>. Acesso em 05 Jun. /2013.

DE SOUSA, A. M. L.; CASTRO, N. M. R.; CANALES, F. A.; LOUZADA, J. A. S.; VITORINO, M. I. ; DE SOUZA, E. B. Multiscale variability of the evapotranspiration in eastern Amazônia. Atmospheric Science Letters, v. 11. 2010, p. 192-198.

DE SOUZA, E. B.; ROCHA, E. J. P. Climatologia, variabilidade e tendências do clima atual na Amazônia e em cenários futuros de mudanças climáticas. In: VIEIRA, I. C. G.; TOLEDO, P. M.; SANTOS JR. R. A. O. (org.) Ambiente e sociedade na Amazônia: uma abordagem interdisciplinar. 1 ed. Rio de Janeiro: Garamond, 2014, p.295-314.

EMPRESA BRASILEIRA DE PESQUISA AGROPECUÁRIA - EMBRAPA. Sistema brasileiro de classificação de solos. Centro Nacional de Pesquisa de Solos (Brasil). Serviço de Produção de Informação. Brasília: EMBRAPA, SPI. 2009, 412 p.

GOEL, N. S. 1989. Models of Vegetation Capony Reflectance and Their Use. In: Estimation of Biophysical Parameters from Reflectance Data. Remote Sensing. Vol. 4, p3-18 José dos Campos/ INPE.

HUDSON, W.D.; RAMM, C.W. Correct formulation of the kappa coefficient of agreement. Photogrammetric Engineering e Remote Sensing. Maryland, v.53, n.4. 1987, p.421-2.

INSTITUTO BRASILEIRO DE GEOGRAFIA E ESTATÍSTICA - IBGE. Base cartográfica digital das cidades - escala 1:100.000. Rio de Janeiro - RJ. Disponível em: <http://www.ibge.gov.br/cidadesat/default. php.> Acesso em: 07/dez/ 2014.

Manual técnico da vegetação brasileira. Rio de Janeiro - RJ. 2012, 271p. Disponível em: <ftp://geoftp. ibge.gov.br/documentos/recursos_naturais/ manuais_tecnicos/manual_tecnico_vegetacao_brasileira.pdf.> Acesso em: 07/dez/ 2014.

LAMEIRA, W. J. de; VIEIRA, I. C. G.; TOLEDO, P. M de. Panorama da Sustentabilidade na Fronteira Agrícola de Bioenergia na Amazônia. Sustentabilidade em Debate Brasília, v. 6, n. 2, p. 193-210, mai/ago 2015. Disponível em: < http://periodicos.unb. br/index.php/sust/article/viewFile/12696/11506> Acesso em: jun./ 2015. 
MINISTÉRIO DO DESENVOLVIMENTO AGRÁRIO - MDA. Programa Nacional de Produção e Uso de Biodiesel: Inclusão social e desenvolvimento territorial. Relatório técnico. Secretaria da Agricultura familiar. 2010, 56p.

MYUNG, I. J. Tutorial on maximum likelihood estimation. Journal of Mathematical Psychology n. 47. 2003, p. 90-100. Disponível em: < http://times.cs.uiuc.edu/ course/410/note/mle.pdf.> Acesso em: Dez. /2014.

MONTEIRO, K. F. G. Análise de indicadores de sustentabilidade socioambiental em diferentes sistemas produtivos com palma de óleo no estado do Pará.

2013. 205f. Tese (Doutorado em Ciências Agrárias) - Universidade Federal Rural da Amazônia, Belém, 2013.

MONTEIRO, M. de A.; COELHO, M. C.; BARBOSA, J. M (Orgs.). Atlas socioambiental: municípios de Tomé-Açu, Ipixuna do Pará, Paragominas e Ulianópolis. Belém: NAEA, 2009, 463 p.

NAHUM, J. S.; MALCHER, A. T. C. Dinâmicas territoriais do espaço agrário na Amazônia: a dendeicultura na microrregião de Tomé-Açu (PA). Revista Confins, 2012. Disponível em: <http://confins.revues.org/7793>. Acesso em: 27 jun. 2013.

NAHUM, J. S.; SANTOS, C. B. Dos. Uma interpretação geográfica da dendeicultura na Amazônia paraense. Revista da Associação Nacional de Pós-graduação e Pesquisa em Geografia - ANPEGE. p.309-331, V.11, n.15, jan-jun. 2015. Disponível em: < http:/ / anpege.org.br/revista/ojs-2.4.6/index.php/anpege08/article/viewFile/423/ pdf> Acesso em Out./2013.

QUEIROZ FILHO, A. P. de; MARTINELLI, M. O trabalho de campo em geografia: uma abordagem teórico-metodológica. Boletim Paulista de Geografia, São Paulo, n. 87. 2007, p. 7-43.

ROSENFIELD, G. H.; FITZPTRIK-LINS, K. A Coefficent of Agreement as Measure of Thematic Classification Accuracy. Photogrammetric Engineering and Remote Sensing. Vol. 52. 1986, p. 223-227.

USGS (GEOLOGICALSURVEY/ SERVIÇODELEVANTAMENTO GEOLÓGICO AMERICANO). Imagens orbitais digitais gratuitas do satélite Landsat-8: data de passagem 04/08/2013 EUA. Disponível em < http://landsat.usgs.gov>. Acesso em 21 ago./2014.

TOMLIN, D. Geographic information systems and Cartographic Modeling. Prentice Hall, New York, 1990.

SILVA, J. C. dos; HOMMA, A, K. O.; SENA, A. L. Dos S.; GOMES JR, R.A.; MENEZES,A. J. E.; MONTEIRO, K. F. G. Desempenho socioeconômico do sistema produtivo familiar de dendê em Moju, estado do Pará. Belém, PA: Embrapa Amazônia Oriental, 2014. 36 p. Boletim de pesquisa e desenvolvimento. Embrapa Amazônia Oriental. 
